\title{
Black holes and universality classes of critical points
}

\author{
Pavel Kovtun and Adam Ritz
}

Department of Physics and Astronomy, University of Victoria, Victoria, BC, V8P 5C2 Canadd

(Dated: January 2008)

\begin{abstract}
We argue that there exists an infinite class of conformal field theories in diverse dimensions, having a universal ratio of the central charge $c$ to the normalized entropy density $\tilde{c}$. The universality class includes all conformal theories which possess a classical gravity dual according to the AdS/CFT correspondence. From the practical point of view, the universality of $c / \tilde{c}$ provides an explicit test which can be applied to determine whether a given critical point may admit a dual description in terms of classical gravity.
\end{abstract}

Introduction:-Many useful quantum field theories are either conformal field theories (CFT), or relevant deformations of a CFT. Among other things, such field theories describe the interactions of all known elementary particles, the scaling behaviour near critical points in statistical mechanics, and the degrees of freedom on the string worldsheet.

There are several procedures via which one can arrive at an interacting CFT. One way is by a critical limit of a statistical-mechanical model (or, more generally, by following the renormalization group flow to the endpoint) [1]. Another possible way to arrive at a CFT is to start with a Lagrangian formulation of a classically conformal theory, and use extra symmetry (such as supersymmetry) to argue that the quantum theory must be conformally invariant as well 22]. A prime example of such a CFT is the $\mathcal{N}=4$ supersymmetric Yang-Mills theory in $3+1$ dimensions. Yet another way to arrive at a CFT is through a theory of (quantum) gravity in Anti-de Sitter (AdS) space, and the AdS/CFT correspondence [3, 4, [5, 6].

In the recent years, there has been a renewed interest in CFTs due to their emergence in quantum critical phenomena; in particular, relativistic CFTs were proposed as the relevant language to describe critical quantum magnets [7]. In turn, understanding CFTs in the language of classical gravity has been useful in the studies of quantum critical transport [8]. This motivates further understanding of the relation of the AdS/CFT correspondence to quantum criticality.

In the context of the AdS/CFT correspondence, a CFT in $d$ dimensions has a dual description in terms of string/M theory on $A d S_{d+1} \times X$, for some compact space $X$. The cosmological constant of $A d S_{d+1}$ takes discrete values, determined by the quantized fluxes of various fields on $X$. It was realized some time ago [9] that the number of such $A d S_{d+1} \times X$ solutions in string theory is enormous: for $d=3$ alone, different choices of $X$ plus various fluxes give rise to an estimated $10^{500}$ solutions - the so-called string landscape [10]. It is believed that every such compactification gives rise to a CFT; in other words, string theory allows one to describe roughly $10^{500}$ different CFTs, or $10^{500}$ different universality classes in three dimensions. It is not unreasonable to ask: does such a multitude of CFTs include any of the real-world critical points? For example, is there a string dual description in $A d S_{4}$ of the simplest liquid-gas critical point (the Ising CFT in $d=3$ )? A simpler question is whether there are interesting fixed points in statistical-mechanical models whose description (in a suitable large- $N$ limit) may be captured by Einstein gravity in $A d S$. It is the purpose of this Letter to propose a criterion for whether a given CFT may have a dual gravitational description within the AdS/CFT correspondence.

Central charge and entropy:- The central charge $c$ is a fundamental quantity which characterizes a given CFT. Given the standard definition of the energy-momentum tensor $T_{\mu \nu}$, we define $c$ via Cardy's [11] parametrization of the two-point function in $d$ spacetime dimensions,

$$
\begin{aligned}
G_{\mu \nu, \alpha \beta}(x) \equiv & \left\langle T_{\mu \nu}(x) T_{\alpha \beta}(0)\right\rangle \\
= & \frac{1}{S_{d}^{2}} \frac{c}{\left(x^{2}\right)^{d}}\left[\left(\delta_{\mu \alpha}-2 \frac{x_{\mu} x_{\alpha}}{x^{2}}\right)\left(\delta_{\nu \beta}-2 \frac{x_{\nu} x_{\beta}}{x^{2}}\right)\right. \\
& +\left(\delta_{\mu \beta}-2 \frac{x_{\mu} x_{\beta}}{x^{2}}\right)\left(\delta_{\nu \alpha}-2 \frac{x_{\nu} x_{\alpha}}{x^{2}}\right) \\
& \left.-\frac{2}{d} \delta_{\mu \nu} \delta_{\alpha \beta}\right],
\end{aligned}
$$

with $S_{d}=2 \pi^{d / 2} / \Gamma(d / 2)$ the volume of the unit $(d-1)$ sphere.

The value of $c$ is one way to measure the number of degrees of freedom in a CFT. A more conventional way to measure the number of degrees of freedom in any system is to heat it up and study its entropy as a function of temperature. ${ }^{1}$ In a $d$ dimensional CFT, the entropy density $s=S / V$ (we take $V \rightarrow \infty$ ) is proportional to $T^{d-1}$ because $T$ is the only dimensionful parameter. The dimensionless proportionality coefficient measures the number

\footnotetext{
1 The formulas are written for $d$ Euclidean dimensions, but the discussion can be repeated almost verbatim for Minkowski CFTs with $d-1$ dimensional space plus time. For a Euclidean theory, heating up means compactifying one of the $d$ dimensions with periodic boundary conditions for bosons and anti-periodic for fermions. The temperature $T$ is then the inverse compactification radius.
} 
of degrees of freedom in the system. We write this relation as

$$
s=\tilde{c} \frac{\Gamma(d / 2)^{3}}{4 \pi^{d / 2} \Gamma(d)}\left(\frac{4 \pi}{d}\right)^{d} \frac{d-1}{d+1} T^{d-1},
$$

which defines the "normalized entropy density" $\tilde{c}$. The dimension-dependent normalization factor is simply a convenient convention. In $d=2$ dimensions, $c$ and $\tilde{c}$ are related by conformal symmetry 12, 13], so that (given our conventions)

$$
d=2 \quad \Longrightarrow \quad c=\tilde{c},
$$

or $s=\pi c T / 3$. It is essentially this relation between $c$ and $\tilde{c}$ in two-dimensional CFTs which allowed for the string theoretic calculation of black hole entropy [14]. In three dimensions, the ratio $c / \tilde{c}$ has been computed in the critical $O(N)$ sigma model at large $N$ [15], with the result:

$$
\frac{c}{\tilde{c}}=\frac{5 \pi^{4}}{2^{4} 3^{3} \zeta(3)}=0.937909 \ldots
$$

However, a general relation between $c$ and $\tilde{c}$ in dimension three and higher is unknown, and remains an important open problem. We will now show that in all $d \geqslant 3$ dimensional CFTs which admit a dual gravitational description via the AdS/CFT correspondence, the central charge is equal to the normalized entropy density,

$$
\mathrm{AdS} / \mathrm{CFT} \Longrightarrow c=\tilde{c} .
$$

This is the main result of the paper to be derived below, and will be discussed further at the end of this Letter.

Entropy in AdS/CFT:-Within the AdS/CFT correspondence, a $d$-dimensional CFT at finite temperature is described by a $D$-dimensional black hole metric

$$
d s^{2}=\frac{r^{2}}{L^{2}}\left(f(r) d x_{0}^{2}+d \mathbf{x}^{2}\right)+\frac{L^{2}}{r^{2}} \frac{d r^{2}}{f(r)}+L_{X}^{2} d s_{X}^{2} .
$$

Here $x=\left(x_{0}, \mathbf{x}\right)$ is $d$-dimensional, $L^{2}$ sets the value of the cosmological constant, $f(r)=1-\left(r_{0} / r\right)^{d}$ where $r=r_{0}$ is the horizon, and $d s_{X}^{2}$ is the metric on $X$. The temperature of the CFT is $T=r_{0} d /\left(4 \pi L^{2}\right)$, and the zerotemperature limit corresponds to $r_{0} \rightarrow 0$. The entropy is proportional to the $D-2$ dimensional area of the horizon, $S=A_{D-2} / 4 G_{N}$ where $G_{N}$ is the $D$-dimensional Newton's constant. Dividing by the (infinite) $(d-1)$-volume $V$, one finds for the entropy density,

$$
s=\frac{1}{4 G_{N}^{(d+1)}}\left(\frac{4 \pi L}{d}\right)^{d-1} T^{d-1},
$$

where the $(d+1)$-dimensional Newton's constant is $1 / G_{N}^{(d+1)}=L_{X}^{D-(d+1)} \operatorname{Vol}(X) / G_{N}$.
Central charge in AdS/CFT:- To find the central charge $c$ in dimension $d \geqslant 3$, one can use either the position or momentum space representation of the correlation function (11) (at $T=0$ ). A convenient momentum space representation is [16]

$$
\begin{aligned}
G_{\mu \nu, \alpha \beta}(k)= & \left(P_{\mu \alpha} P_{\nu \beta}+P_{\mu \beta} P_{\nu \alpha}\right. \\
& \left.-\frac{2}{d-1} P_{\mu \nu} P_{\alpha \beta}\right) G\left(k^{2}\right),
\end{aligned}
$$

where $P_{\mu \nu}=\delta_{\mu \nu}-k_{\mu} k_{\nu} / k^{2}$. The central charge is related to $G\left(k^{2}\right)$ by

$$
\frac{c}{\left(x^{2}\right)^{d}}=\frac{d+1}{d-1} S_{d}^{2} \int \frac{d^{d} k}{(2 \pi)^{d}} e^{i k x} G\left(k^{2}\right) .
$$

Choosing $k$ along $x_{i}$ with $i \neq 1$ or 2 , one has $G_{12,12}(k)=$ $G\left(k^{2}\right)$, and therefore it suffices to evaluate $G_{12,12}$ to find the central charge. According to the AdS/CFT prescription, $G_{12,12}$ is given by the second variation of the gravitational action with respect to the boundary value of the metric perturbation $h_{12}$. The $h_{12}$ perturbation decouples from all other perturbations, and obeys the equation of motion coming from the action of a massless scalar in the $A d S_{d+1}$ background,

$$
\begin{aligned}
& \int d^{d+1} x \sqrt{g}(R-\Lambda)+2 \int d^{d} x \sqrt{g_{B}} K \\
& =-\frac{1}{2} \int d^{d+1} x \sqrt{g} g^{\mu \nu} \partial_{\mu} \phi \partial_{\nu} \phi+\ldots
\end{aligned}
$$

Here $\phi \equiv h_{2}^{1}$ (we require $d \geqslant 3$ ), the cosmological constant is $\Lambda=-d(d-1) / L^{2}$, the second term in the action is the standard Gibbons-Hawking boundary term, and contact boundary terms are dropped on the righthand side of (10). The two-point correlation function for the massless scalar can be evaluated using the standard AdS/CFT prescription either in momentum space, following [4], or in position space [5, 17]. Restoring the overall factor of $1 / 16 \pi G_{N}^{(d+1)}$ in front of the action (10), one finds for the central charge,

$$
c=\frac{d+1}{d-1} \frac{L^{d-1}}{4 \pi G_{N}^{(d+1)}} \frac{\Gamma(d+1) \pi^{d / 2}}{\Gamma(d / 2)^{3}},
$$

in agreement with [18]. Comparing this to $\tilde{c}$ as found from (7), we arrive at our main result in Eq. (5), $c / \tilde{c}=1$. For $\mathcal{N}=1$ supersymmetric CFTs in $d=4$, this relation was discussed earlier in Ref. [19].

Discussion:-What we have shown is that every CFT in dimension $d \geqslant 3$ which has an $A d S_{d+1}$ gravity dual description must have a central charge equal to the normalized entropy density. More precisely, this equality should hold up to corrections which vanish in the limit in which a classical gravitational description in $A d S$ is valid, e.g. at large $N$ and large 't Hooft coupling for $\mathcal{N}=4$ SYM and 
variants thereof. Furthermore, we should note that the reduction in (10) clearly fails for $d \leqslant 2$ as Einstein gravity then has no propagating degrees of freedom; nonetheless conformal invariance is sufficient in $d=2$ to ensure $c=\tilde{c}$ for all CFTs regardless of whether they admit a dual gravitational description in $A d S_{3}$. It is natural to ask if there are "conventional" CFTs which also have $c=\tilde{c}$. It is obvious that $c$ cannot be equal to $\tilde{c}$ in non-interacting (or weakly interacting) theories when $d$ is odd. This is because in a free theory $\tilde{c}$ is proportional to $\zeta(d)$, while $c$ contains no such irrational factors. In even dimensions, this is no longer the case and one may wonder whether free theories may exist which satisfy $c=\tilde{c}$. We can test this in four dimensions where, for a free theory with $n_{v}$ vector, $n_{f}$ fermionic and $n_{s}$ scalar degrees of freedom, we find that

$$
\left(\frac{c}{\tilde{c}}\right)_{4 d}^{\text {free }}=\frac{3}{8} \frac{n_{s}+\frac{3}{2} n_{f}+12 n_{v}}{n_{s}+\frac{7}{8} n_{f}+2 n_{v}},
$$

so that $3 / 8 \leqslant c / \tilde{c} \leqslant 9 / 4$. Note that $c / \tilde{c}=3 / 4$ for the free limit of $\mathcal{N}=4 \mathrm{SYM}$ consistent with the known difference of the entropy (and thus $\tilde{c}$ ) at strong and weak coupling [6]. Importantly, we observe from this relation that free theories do exist which satisfy $c=\tilde{c}$, provided they contain vector degrees of freedom, such that $2 n_{s}+n_{f}=8 n_{v}$; the free limit of QED with two flavors is a simple example. Thus we conclude that, at least in $d=4$, the relation $c=\tilde{c}$ is necessary but not sufficient for a given CFT to possess a gravity dual. However, when $d$ is odd, it is tempting to conjecture that the condition $c=\tilde{c}$ is not only necessary, but also sufficient for a given CFT to have a gravity dual.

Let us also point out that a criterion similar to (5) is known for $d=4$ CFTs. In four dimensions, there are two central charges, commonly denoted by $c$ and $a$ (which characterize the response to two different curvature invariants when the CFT is placed in curved space). It turns out that the AdS/CFT formulation implies (assuming a certain choice of normalization) that $c=a$ in the limit that the classical gravitational description is valid [20, 21]. The condition $c=a$ has been considered as a means of classification 22] and is necessary for a $d=4$ CFT to have a dual gravity description in the appropriate large- $N$ limit. However, it also is not sufficient because there are examples (such as $\mathcal{N}=4$ super Yang-Mills) where $c=a$ holds at both strong and weak coupling [20, 21], while the gravitational description is only valid at strong coupling. The condition $c=\tilde{c}$ is clearly stronger than $c=a$ because $i$ ) it applies in any dimension $d \geqslant 3$, not just in $d=4$, and perhaps more importantly ii) it is in principle capable of making a distinction between strongly and weakly coupled theories because $\tilde{c}$ is not protected by supersymmetry.

In physical terms, the condition $c / \tilde{c}=1$ is a realspace counterpart of the relation $\eta / s=1 / 4 \pi$, where $\eta$ is the shear viscosity of any field theory with a dual $A d S$ gravity description [23, 24]. Indeed, the Kubo formula for $\eta$ relates the shear viscosity to the thermal real-time correlation function $G_{12,12}(k)$ at small timelike momentum. On the other hand, the central charge is related to $G_{12,12}(k)$ at large spacelike momentum, where the effects of temperature do not matter. This analogy can be made more transparent if we trade $c$ for the absorption crosssection $\sigma(\omega)$ (in $D$-dimensional Planck units) for graviton scattering by the appropriate gravitational background [6] and contrast $\eta / s$ with the high-frequency limit of $\sigma(\omega) / s \propto c / \tilde{c}$ at temperature $T .{ }^{2}$ Indeed, the dual gravitational perturbation $\phi=h_{2}^{1}$ behaves as a massless scalar in Eq. (10), regardless of the temperature [23]. However, within AdS/CFT, the ratio $\eta / s$ apparently defines a wider class than $c / \tilde{c}$ because $\eta / s=1 / 4 \pi$ for both CFTs and relevant deformations of CFTs. In addition, $\eta / s=1 / 4 \pi$ applies universally for any $d \geqslant 3$, without dimension-dependent normalization factors. However, from the point of view of finding a possible gravity dual for a given CFT, the condition $c / \tilde{c}=1$ has a significant advantage over $\eta / s=1 / 4 \pi$ because it involves only equilibrium quantities which are easier to compute than real-time response functions.

Focusing on 3-dimensional systems, relevant to realworld critical points, we find it interesting that the large$N$ result (4) for $c / \tilde{c}$ in the $O(N)$ model is numerically very close to one, the value required for the existence of a gravity dual. With regard to the proposal of Klebanov and Polyakov 25] - that the large- $N$ dual is a higher-spin gauge theory in AdS - this result amounts to a prediction for the bulk spin-two sector and implies a (small) quantitative difference with pure Einstein gravity. At the opposite end of the spectrum, the critical Ising model corresponds to $N=1$ and it would clearly be interesting to see if $1 / N$ corrections [26], known to be generically rather large [27], were to modify Eq. (4) bringing $c / \tilde{c}$ closer to one $(c / \tilde{c}$ could also be computed directly at the Wilson-Fisher fixed point using the epsilon expansion). However, the possibility of $c / \tilde{c}=1$ in this case would go against the general expectation that the critical Ising model has too few degrees of freedom to possess a classical gravity dual. This is because every CFT with a classical gravity dual has a finite-volume phase transition [28] as a function of $T$, and therefore must have an infinite number of degrees of freedom. Nevertheless, one hopes that suitable limits exist in which these CFTs are close to real-world examples. It would be interesting to investigate more generally the corrections to $c / \tilde{c}=1$ arising from quantum corrections to classical gravity.

2 The fact that $c / \tilde{c}=1$ implies that $\sigma /\left(s G_{N}\right)=f(d)(\omega / T)^{d-1}$, where the constant $f(d)$ is purely a function of the spacetime dimension of the CFT. For $d=3,4,6$, the constant $f(d)$ can be found from the $A d S$ duals of M2, D3, and M5 branes, using the results [6] for $\sigma(\omega)$. 
Finally, we have seen that $A d S$ gravity provides us with a multitude of non-Gaussian fixed points with exactly the same value of $c / \tilde{c}$. However, it is far from obvious how these fixed points are related to each other; in particular, they have different symmetries. While Monte Carlo simulations should be tractable in many cases, we are not aware of numerical results for $c / \tilde{c}$ at non-Gaussian fixed points in any three-dimensional lattice model. Thus, going beyond the classical gravity approximation, as a related question we may ask: are there non-Gaussian fixed points in three dimensions that share the same value of $c / \tilde{c}$ (not necessarily equal to one)? A positive answer would suggest a novel notion of universality, which is not related to symmetry, but may be related to (quantum) gravity in Anti-de Sitter space.

We are grateful to Subir Sachdev for helpful discussions, and to Sean Hartnoll, Chris Herzog, Anastasios Petkou, Kostas Skenderis, Tadashi Takayanagi, and Edward Witten for comments on the manuscript. This work was supported in part by NSERC of Canada.

* Electronic address: pkovtun@uvic.ca, aritz@uvic.ca

[1] K. G. Wilson and J. B. Kogut, Phys. Rept. 12, 75 (1974).

[2] See e.g. R. G. Leigh and M. J. Strassler, Nucl. Phys. B 447, 95 (1995) arXiv:hep-th/9503121.

[3] J. M. Maldacena, Adv. Theor. Math. Phys. 2, 231 (1998) [Int. J. Theor. Phys. 38, 1113 (1999)] arXiv:hep-th/9711200.

[4] S. S. Gubser, I. R. Klebanov and A. M. Polyakov, Phys. Lett. B 428, 105 (1998) arXiv:hep-th/9802109.

[5] E. Witten, Adv. Theor. Math. Phys. 2, 253 (1998) arXiv:hep-th/9802150.

[6] For a review, see I. Klebanov's TASI'99 lectures, arXiv:hep-th/0009139.

[7] For a review, see S. Sachdev, Nature Physics 4, 173 (2008) arXiv:0711.3015 [cond-mat.str-el]].
[8] C. P. Herzog, P. Kovtun, S. Sachdev and D. T. Son, Phys. Rev. D 75, 085020 (2007) arXiv:hep-th/0701036.

[9] R. Bousso and J. Polchinski, JHEP 0006, 006 (2000) arXiv:hep-th/0004134;

[10] For a review, see M. R. Douglas and S. Kachru, Rev. Mod. Phys. 79, 733 (2007) arXiv:hep-th/0610102.

[11] J. L. Cardy, Nucl. Phys. B 290, 355 (1987).

[12] H. W. J. Bloete, J. L. Cardy and M. P. Nightingale, Phys. Rev. Lett. 56, 742 (1986).

[13] I. Affleck, Phys. Rev. Lett. 56, 746 (1986).

[14] A. Strominger and C. Vafa, Phys. Lett. B 379, 99 (1996) arXiv:hep-th/9601029.

[15] S. Sachdev, Phys. Lett. B 309, 285 (1993) arXiv:hep-th/9305131.

[16] P. K. Kovtun and A. O. Starinets, Phys. Rev. D 72, 086009 (2005) arXiv:hep-th/0506184.

[17] D. Z. Freedman, S. D. Mathur, A. Matusis and L. Rastelli, Nucl. Phys. B 546, 96 (1999) arXiv:hep-th/9804058.

[18] H. Liu and A. A. Tseytlin, Nucl. Phys. B 533, 88 (1998) arXiv:hep-th/9804083.

[19] T. Nishioka and T. Takayanagi, Phys. Rev. D 76, 044004 (2007) arXiv:hep-th/0702194.

[20] M. Henningson and K. Skenderis, JHEP 9807, 023 (1998) arXiv:hep-th/9806087.

[21] S. S. Gubser, Phys. Rev. D 59, 025006 (1999) arXiv:hep-th/9807164.

[22] D. Anselmi, Phys. Lett. B 476, $182 \quad$ (2000) arXiv:hep-th/9908014.

[23] P. Kovtun, D. T. Son and A. O. Starinets, Phys. Rev. Lett. 94, 111601 (2005) arXiv:hep-th/0405231.

[24] A. Buchel, Phys. Lett. B 609, 392 (2005) arXiv:hep-th/0408095.

[25] I. R. Klebanov and A. M. Polyakov, Phys. Lett. B 550, 213 (2002) arXiv:hep-th/0210114.

[26] A. C. Petkou, Phys. Lett. B 359, 101 (1995) arXiv:hep-th/9506116.

[27] See e.g. J. Zinn-Justin, "Quantum field theory and critical phenomena," Oxford, 1989.

[28] E. Witten, Adv. Theor. Math. Phys. 2, 505 (1998) arXiv:hep-th/9803131. 